\title{
Projection Neurons in Lamina III of the Rat Spinal Cord Are Selectively Innervated by Local Dynorphin-Containing Excitatory Neurons
}

\author{
Najma Baseer, ${ }^{1}$ Erika Polgár, ${ }^{1}$ Masahiko Watanabe, ${ }^{2}$ Takahiro Furuta, ${ }^{3}$ Takeshi Kaneko, ${ }^{3}$ and Andrew J. Todd ${ }^{1}$ \\ ${ }^{1}$ Spinal Cord Group, Institute of Neuroscience and Psychology, University of Glasgow, Glasgow G12 8QQ, United Kingdom; 2 Department of Anatomy, \\ Hokkaido University School of Medicine, Sapporo 060-8638, Japan; and '3epartment of Morphological Brain Science, Graduate School of Medicine, Kyoto \\ University, Kyoto 606-8501, Japan
}

\begin{abstract}
Large projection neurons in lamina III of the rat spinal cord that express the neurokinin 1 receptor are densely innervated by peptidergic primary afferent nociceptors and more sparsely by low-threshold myelinated afferents. However, we know little about their input from other glutamatergic neurons. Here we show that these cells receive numerous contacts from nonprimary boutons that express the vesicular glutamate transporter 2 (VGLUT2), and form asymmetrical synapses on their dendrites and cell bodies. These synapses are significantly smaller than those formed by peptidergic afferents, but provide a substantial proportion of the glutamatergic synapses that the cells receive (over a third of those in laminae I-II and half of those in deeper laminae). Surprisingly, although the dynorphin precursor preprodynorphin (PPD) was only present in 4-7\% of VGLUT2 boutons in laminae I-IV, it was found in $58 \%$ of the VGLUT2 boutons that contacted these cells. This indicates a highly selective targeting of the lamina III projection cells by glutamatergic neurons that express PPD, and these are likely to correspond to local neurons (interneurons and possibly projection cells). Since many PPD-expressing dorsal horn neurons respond to noxious stimulation, this suggests that the lamina III projection cells receive powerful monosynaptic and polysynaptic nociceptive input. Excitatory interneurons in the dorsal horn have been shown to possess $I_{A}$ currents, which limit their excitability and can underlie a form of activity-dependent intrinsic plasticity. It is therefore likely that polysynaptic inputs to the lamina III projection neurons are recruited during the development of chronic pain states.
\end{abstract}

\section{Introduction}

Despite the importance of the spinal dorsal horn in processing sensory information, we know relatively little about its neuronal circuitry, largely because of the difficulty of defining functional populations among both projection neurons and interneurons (Todd, 2010). We have identified a distinctive population of lamina III projection neurons that express the neurokinin 1 receptor (NK1r) and have axons that ascend through the anterolateral tract to the brainstem and thalamus (Marshall et al., 1996; Todd et al., 2000; Al-Khater et al., 2008). These cells have extensive dorsal dendrites that are densely innervated by substance P-containing nociceptive primary afferents (Lawson et al., 1997; Naim et al., 1997), and also receive a modest synaptic input from myelinated low-threshold afferents (Naim et al., 1998).

The great majority of dorsal horn neurons are interneurons, with axons that arborize locally. Around one-third of those in

\footnotetext{
Received June 6, 2012; revised July 6, 2012; accepted July 12, 2012

Author contributions: N.B. and A.J.T. designed research; N.B., E.P., and A.J.T. performed research; N.B. and A.J.T. analyzed data; N.B., E.P., M.W., T.F., T.K., and A.J.T. wrote the paper.

Financial support from the Wellcome Trust and the Khyber Medical University, Pakistan, is gratefully acknowledged. We thank R. Kerr and C. Watt for expert technical assistance and Dr Z. Puskár for advice.

The authors declare no competing financial interests.

Correspondence should be addressed to Andrew J. Todd, Spinal Cord Group, West Medical Building, University of Glasgow, University Avenue, Glasgow G12 80Q, UK. E-mail: andrew.todd@glasgow.ac.uk.

DOI:10.1523/JNEUROSCI.2707-12.2012

Copyright $\odot 2012$ the authors $\quad 0270-6474 / 12 / 3211854-10 \$ 15.00 / 0$
}

laminae I-III are inhibitory, using GABA and/or glycine as their fast transmitter, while the remainder are glutamatergic and contain high levels of vesicular glutamate transporter 2 (VGLUT2) in their axonal boutons (Todd et al., 2003, 2010; Yasaka et al., 2010). Attempts to classify interneurons based on morphological criteria have identified certain types (e.g., islet, central, vertical, and radial cells in lamina II) (Grudt and Perl, 2002; Heinke et al., 2004; Graham et al., 2007; Yasaka et al., 2007), but this approach has met with limited success because many cells cannot be assigned to a specific morphological class (Yasaka et al., 2010). As an alternative strategy, we have identified several neurochemically defined populations among the inhibitory interneurons in this region (Tiong et al., 2011), and have shown that some of these are selective in their postsynaptic targets. For example, GABAergic neurons that express neuropeptide $\mathrm{Y}$ (NPY) preferentially innervate the lamina III NK1r ${ }^{+}$projection neurons (Polgár et al., 1999, 2011). However, much less is known about the organization of synaptic circuits involving excitatory interneurons. Although electrophysiological studies involving paired recordings have identified glutamatergic synapses between vertical cells and lamina I projection neurons (Lu and Perl, 2005), there is currently no evidence for selective innervation of projection cells by specific types of excitatory neuron in the dorsal horn.

The first aim of this study was to compare the frequency of excitatory input to the lamina III NK1 $\mathrm{r}^{+}$projection neurons from nonprimary VGLUT2-immunoreactive boutons (which 
Table 1. Antibodies used in this study

\begin{tabular}{llll}
\hline Antibody & Species & Dilution & Source \\
\hline NK1r & Rabbit & $1: 1000$ or 1:10,000 & Sigma Aldrich \\
VGLUT2 & Goat & $1: 500$ & M Watanabe \\
VGLUT2 & Guinea pig & $1: 1000$ & Millipore \\
PPD & Guinea pig & $1: 1000$ or 1:5000 & T Kaneko \\
PPD & Rabbit & $1: 10,000$ & T Kaneko \\
CGRP & Guinea pig & $1: 10,000$ & Bachem \\
CGRP & Sheep & $1: 5000$ & Enzo Life Sciences \\
VGLUT1 & Guinea pig & $1: 5000$ & Millipore \\
\hline
\end{tabular}

are likely to originate from local neurons) with that from primary afferents. Some excitatory neurons in dorsal horn express the dynorphin precursor preprodynorphin (PPD) (Marvizón et al., 2009; Sardella et al., 2011a) and preliminary observation showed that the lamina III projection neurons received numerous contacts from VGLUT2 boutons that contained PPD. The second aim was therefore to determine whether this represented a selective innervation by dynorphin-containing cells. We have shown that there is a wide variation in the sizes of glutamatergic synapses on the lamina III projection cells (Todd et al., 2009), and the third aim was to test the hypothesis that synapses formed by peptidergic primary afferents were larger than those from glutamatergic dorsal horn neurons.

\section{Materials and Methods}

Animals and tissue processing. Experiments were approved by the Ethical Review Process Applications Panel of the University of Glasgow and were performed in accordance with the UK Animals (Scientific Procedures) Act 1986.

Eight adult male Wistar rats (300-390 g; Harlan) were deeply anesthetized with pentobarbitone (300 mg, i.p.) and perfused through the left ventricle with a fixative containing $4 \%$ freshly depolymerized formaldehyde (six rats) or $4 \%$ formaldehyde/0.2\% glutaraldehyde (two rats). Mid-lumbar (L3-L5) segments were removed and cut into 60- $\mu$ m-thick parasagittal sections with a vibrating microtome. Sections were treated for $30 \mathrm{~min}$ with $50 \%$ ethanol and then processed for immunocytochemistry, as described below. In all cases incubations in primary antibodies were at $4^{\circ} \mathrm{C}$ for $3 \mathrm{~d}$ and those in secondary antibodies were overnight. Antibodies were diluted in PBS that contained $0.3 \%$ Triton X-100, unless otherwise stated, and details of the sources and concentrations are given in Table 1. All secondary antibodies were raised in donkey and were species specific. Fluorescent secondary antibodies were conjugated to Rhodamine Red, DyLight 649 (1:100; both from Jackson ImmunoResearch) or Alexa 488 (1:500; Invitrogen). Secondary antibodies labeled with biotin (1:500) or horseradish peroxidase (HRP; 1:1000, both from Jackson ImmunoResearch) were also used. The biotinylated antibodies were revealed with avidin-Pacific Blue (Invitrogen; 1:1000), or with avidin-HRP (Sigma; 1:1000) followed by either tyramide signal amplification (TSA; tetramethylrhodamine kit; PerkinElmer Life Sciences), or by reaction with 3,3'-diaminobenzidine (DAB) in the presence of hydrogen peroxide. The HRP-labeled secondary antibodies were revealed with TSA. Sections for confocal microscopy were mounted in anti-fade medium and stored at $-20^{\circ} \mathrm{C}$.

Analysis of contacts from VGLUT2-expressing, and primary afferent boutons on to lamina III NK1r cells. VGLUT2 is present at high levels in axonal boutons derived from local excitatory neurons (Todd et al., 2003) and in some of those from descending axons (Llewellyn-Smith et al., 2007; Shrestha et al., 2012). However, it is also seen at very low levels in some myelinated low-threshold mechanoreceptive and peptidergic primary afferents (Todd et al., 2003; Alvarez et al., 2004; Persson et al., 2006), both of which provide synaptic input to NK1r-expressing lamina III projection neurons (Naim et al., 1997, 1998). All of the low-threshold myelinated afferents contain VGLUT1 (Todd et al., 2003; Alvarez et al., 2004), and all peptidergic primary afferents in the rat contain calcitonin gene-related peptide (CGRP) (Ju et al., 1987). To identify VGLUT2 bou- tons that did not originate from either of these types of primary afferent, we initially incubated sections from three of the rats perfused with $4 \%$ formaldehyde in rabbit anti-NK1r, guinea pig anti-CGRP, and goat anti VGLUT2 antibodies (Kawamura et al., 2006), which were revealed with Alexa 488, Pacific blue, and Rhodamine Red, respectively. NK1r immunoreactivity was viewed, and 15 large immunoreactive lamina III neurons with dorsal dendrites that entered the superficial dorsal horn were selected for analysis (five from each rat). These cells were scanned through the full thickness of the section with a Zeiss LSM710 confocal microscope (equipped with Argon multiline, $405 \mathrm{~nm}$ diode, $561 \mathrm{~nm}$ solid-state, and $633 \mathrm{~nm}$ HeNe lasers) through dry $(10 \times, 20 \times)$ and oilimmersion $(63 \times)$ lenses (NAs: $0.3,0.8$, and 1.4, respectively). For all scans obtained with the $63 \times$ lens the pinhole was set to 1.0 Airy unit, and several overlapping fields were scanned (with a $z$-step of $0.5 \mu \mathrm{m}$ ) to include as much of the dendritic tree as was present in the section. These scans were analyzed with Neurolucida for Confocal software (Microbrightfield), and the cell bodies and dendritic trees of the neurons were drawn. Cell body surface areas were measured and the surface areas of dendrites were estimated from their lengths and diameters, based on the assumption that they were cylindrical (Todd et al., 2002). The border between laminae II and III, determined from dark-field scans through the $10 \times$ lens, was plotted onto the drawings and regions of dendrite that lay within the superficial dorsal horn (laminae I and II) were analyzed separately from dendrites and cell bodies in deeper laminae. The locations of contacts from CGRP-immunoreactive varicosities, and from those that were VGLUT2 immunoreactive but lacked CGRP, were plotted onto the cells. The sections were then removed from the slides and re-incubated in guinea pig antibody against VGLUT1, which was also revealed with Pacific blue. They were then rescanned through the $63 \times$ lens and the locations of contacts from VGLUT1-immunoreactive boutons (i.e., those that were only stained with Pacific blue after the second immunoreaction) were plotted. The densities of contacts per $1000 \mu \mathrm{m}^{2}$ of cell surface were then determined for boutons that contained CGRP or VGLUT1, as well as for those that were VGLUT2 immunoreactive and lacked CGRP and VGLUT1.

Analysis of contacts from PPD boutons. To assess coexistence of PPD with VGLUT2 in nonprimary boutons that contact lamina III NK1r projection neurons, we reacted sections from three of the rats that had been fixed with $4 \%$ formaldehyde to reveal NK1r, PPD, VGLUT2, VGLUT1, and CGRP. This was done by initially incubating the sections in rabbit anti-PPD (Lee et al., 1997), goat anti-VGLUT2, and guinea pig antibodies against both CGRP and VGLUT1. The PPD was labeled by the TSA method, while VGLUT2 was revealed with DyLight 649 and both CGRP and VGLUT1 with Pacific blue. The sections were then incubated in rabbit anti-NK1r, which was detected with Alexa 488. Although both PPD and NK1r antibodies were raised in rabbit, the TSA method allowed the PPD antibody to be used at a low concentration $(1: 10,000)$, which was only weakly detected by the Alexa 488-labeled secondary antibody that was used to reveal NK1r. Fifteen large NK1r-immunoreactive lamina III neurons (five from each of the three rats) were selected, scanned with the confocal microscope (as described above), and analyzed with Neurolucida. Initially, only the channels corresponding to Pacific blue, Alexa 488, and DyLight 649 were viewed, and the locations of all contacts from VGLUT2-immunoreactive boutons that lacked CGRP or VGLUT1 were plotted onto the cell bodies and dendrites of the selected neurons. The rhodamine channel was then revealed, and the presence or absence of PPD in each of the VGLUT2 boutons in contact with the NK1r cells was recorded. Again, contacts on dendrites in laminae I-II were analyzed separately from those on cell bodies and dendrites in deeper laminae (III-IV).

The frequency with which PPD was present in VGLUT2 boutons that contacted NK1r-expressing lamina I neurons was also assessed in these sections, to allow comparison with the lamina III cells. Fifteen large lamina I NK1r-immunoreactive neurons (five from each of the three rats) were selected, scanned and analyzed as described above.

To determine the extent to which PPD was expressed in the general population of VGLUT2-immunoreactive nonprimary boutons in superficial and deep laminae, we analyzed sets of confocal scans from two sections from each of the three rats. Each set of scans included an area 
that extended from the dorsal aspect of lamina I to a depth of $300 \mu \mathrm{m}$ below the lamina II/III border. This depth was chosen as it corresponded to the most ventral level at which VGLUT2 contacts were identified on the dendrites of the lamina III NK1r projection neurons. From each scan, 100 boutons that were positive for VGLUT2 and lacked CGRP or VGLUT1 were selected in both superficial and deep regions by placing a square grid $(5 \times 5 \mu \mathrm{m})$ on the images in Neurolucida, as described previously (Polgár et al., 2011). For each region, the eighth optical section in the $z$-series was viewed, and the first bouton was obtained from one of the most dorsal squares. The selection process then continued in a dorsal-to-ventral, followed by left-to-right, direction until 100 boutons had been selected. This was done to ensure that boutons were sampled from all dorsoventral locations within each region. The PPD channel was then viewed and the presence or absence of immunostaining was recorded for each of the selected boutons. Since this selection method will be biased toward boutons that were more extensive in the $z$-axis (Guillery, 2002), we compared $z$-axis lengths of VGLUT2 boutons that contained PPD with those of VGLUT2 ${ }^{+} / \mathrm{PPD}^{-}$boutons by determining the number of optical sections on which each bouton was visible (Polgár et al., 2011).

Combined confocal and electron microscopy. To confirm that synapses were present at contacts between VGLUT2- and PPD-immunoreactive boutons and the lamina III NK1r neurons, we used a combined confocal and electron microscopic method (Naim et al., 1997; Todd, 1997, 2002). Sections from the two rats fixed with glutaraldehyde/formaldehyde underwent one of two different immunostaining protocols. Immunostaining was performed as described above, except that the sections were treated for $30 \mathrm{~min}$ with $1 \%$ sodium borohydride (to minimize nonspecific staining resulting from glutaraldehyde fixation) and antibodies were diluted in PBS that did not contain detergent.

In one case sections were incubated in rabbit anti-NK1r, guinea pig anti-VGLUT2 and sheep anti-CGRP, followed by secondary antibodies conjugated to Alexa 488, Rhodamine Red, and DyLight 649, respectively. The secondary antibody mixture also included biotinylated anti-guinea pig IgG, and the sections were then incubated in avidin-HRP and mounted in anti-fade medium. A large lamina III NK1r-immunoreactive cell was identified and scanned with the confocal microscope to reveal contacts from VGLUT2- and CGRP-containing boutons. The section containing the cell was then removed from the slide and reacted with DAB to reveal the HRP-labeled VGLUT2-immunoreactive profiles. It was then osmicated and embedded in resin. A series of $\sim 130$ ultrathin sections (silver interference color, $\sim 70 \mathrm{~nm}$ thickness) was cut through part of the cell with a diamond knife. The sections were collected in serial order on Formvar-coated slot grids, stained with uranyl acetate and lead citrate, and viewed with a Philips CM100 electron microscope (EM) equipped with a digital camera. The regions of dendrite that appeared in the ultrathin sections were identified based on their location in relation to landmarks (e.g., capillaries) that could be recognized in the confocal image stacks.

Sections from the other rat were incubated in rabbit anti-NK1r, guinea pig anti-PPD (Lee et al., 1997) and goat anti-VGLUT2. The NK1r was revealed with Alexa 488, PPD with TSA-rhodamine, and VGLUT2 with DyLight 649 . The antibody mixture also included biotinylated anti-goat IgG. The sections were then incubated in avidin-HRP and mounted. A large lamina III NK1r neuron was selected and scanned to reveal contacts from PPD- and VGLUT2-immunoreactive boutons. The section containing the cell was then reacted with DAB to reveal VGLUT2, before being osmicated and embedded in resin. Ultrathin sectioning and electron microscopy were performed as described above.

Antibody characterization. The NK1r antibody was raised in rabbit against a peptide corresponding to amino acids 393-407 of the rat NK1r, and recognizes a $46 \mathrm{kDa}$ band in Western blots of rat brain extracts. It has been shown that there is no staining with this antibody in mice in which the NK1r has been deleted (Ptak et al., 2002). The guinea pig VGLUT1 antibody was raised against amino acids $541-560$ of the rat protein, while guinea pig and goat anti-VGLUT2 were raised against peptides corresponding to amino acids 565-582 of rat VGLUT2 and amino acids 550582 of mouse VGLUT2, respectively. The guinea pig antibodies against VGLUT1 and VGLUT2 have been shown to stain identical structures to well characterized rabbit antibodies against the two transporters (Todd et al., 2003), while the goat anti-VGLUT2 detects a single protein band of the appropriate molecular weight ( $60 \mathrm{kDa})$ (Kawamura et al., 2006). The rabbit and guinea pig antibodies against PPD were raised against a peptide corresponding to amino acids 229-248 at the C terminus of rat PPD. They have been shown to label PPD, but not dynorphin or enkephalin, and tissue staining is blocked by pre-absorption of the antibody with the immunizing peptide (Lee et al., 1997). The two CGRP antibodies detect both $\alpha$ and $\beta$ forms of the peptide.

Statistics. Two-way ANOVA on ranks was used to compare densities of different types of bouton on superficial and deep dendrites of the lamina III NK1r cells, and also to compare the percentage of the VGLUT2 boutons contacting these cells that were PPD immunoreactive with those in the general population in both superficial and deep laminae. Differences in PPD expression among VGLUT2 boutons on superficial and deep dendrites of the lamina III cells, and between those on lamina I and lamina III NK1r neurons, were analyzed with Mann-Whitney $U$ tests. This test was also used to compare the sizes of $\mathrm{PPD}^{+}$and $\mathrm{PPD}^{-}$VGLUT2 boutons in the general population. Lengths of different types of synapse were compared by using $t$ tests. $P$ values of $<0.05$ were considered significant.

\section{Results}

\section{Contacts on lamina III NK1r neurons from boutons that contain VGLUT2, VGLUT1, or CGRP}

Sections were initially examined after immunostaining for NK1r, VGLUT2, and CGRP. Large NK1r-immunoreactive lamina III neurons were readily identified in the sagittal sections, and all 15 of those analyzed received many contacts from CGRPimmunoreactive boutons (labeled with Pacific blue). These were particularly frequent on their dorsal dendrites within laminae I-II, and some of them showed very weak VGLUT2 immunostaining. In addition, all of the cells received numerous contacts from boutons that were strongly labeled with the VGLUT2 antibody (Fig. 1). These were seen on both superficial and deep dendrites, and also on cell bodies.

Following incubation in VGLUT1 antibody (which was also revealed with Pacific blue) it was possible to identify VGLUT1immunoreactive profiles, as these had initially been unlabeled and only showed Pacific blue labeling after the second immunoreaction (Fig. 1a). All of the 15 cells received some contacts from VGLUT1-immunoreactive profiles, and these were most numerous on their deep dendrites. Penetration of antibodies was apparently complete, since the numbers of profiles that were immunostained with each of these antibodies were similar throughout the depth of the sections.

Quantitative results for this part of the study are shown in Table 2. The lengths of superficial and deep dendrites analyzed for each cell ranged from 393 to $2189 \mu \mathrm{m}$ (mean $1168 \mu \mathrm{m}$ ) and 119 to $1180 \mu \mathrm{m}$ (mean $560 \mu \mathrm{m}$ ), respectively. Two-way ANOVA on ranks revealed significant differences between the densities of these three types of axonal bouton on superficial (laminae I-II) and deep (laminae III-IV) regions of the cells $(p<0.01)$. Post hoc all-pairwise multiple comparisons (Holm-Sidak method) revealed that the density of contacts from CGRP boutons was significantly higher on superficial dendrites than on deep dendrites and cell bodies (mean 21.1 per $1000 \mu \mathrm{m}^{2}$ for superficial dendrites compared with 11.1 per $1000 \mu \mathrm{m}^{2}$ for deep dendrites and cell bodies, $p<0.01$ ), while the density of VGLUT1 contacts was significantly lower on superficial dendrites than on deep dendrites and cell bodies (means 0.5 and 2.9 per $1000 \mu \mathrm{m}^{2} ; p<0.01$ ). In contrast, the density of contacts from VGLUT2 boutons that lacked CGRP and VGLUT1 did not differ significantly between superficial and deep regions (means 13.6 and 15.5 per $1000 \mu \mathrm{m}^{2}$, $p=0.27$ ). To allow comparison with previous reports (Naim et al., 1997, 1998), we also determined the mean density of contacts 

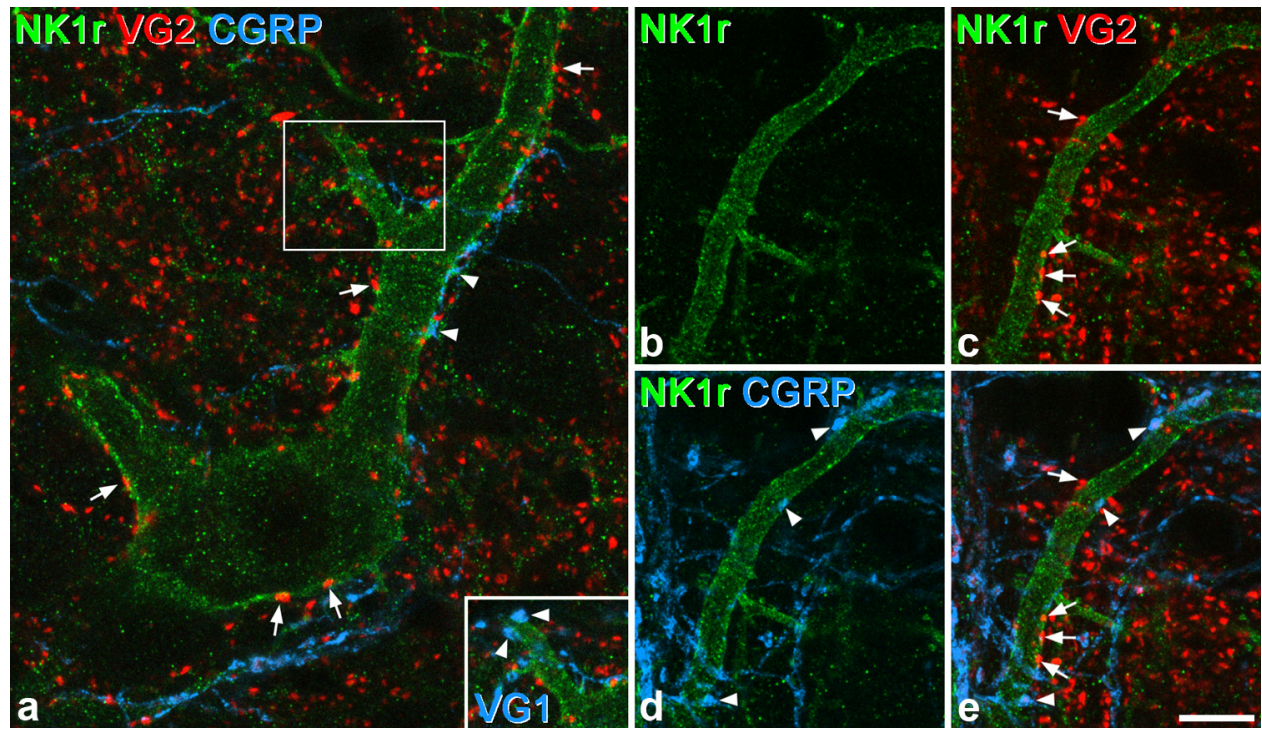

Figure 1. Contacts from axonal boutons that contain VGLUT2 (VG2) or CGRP and a large lamina III neuron that expresses the NK1r. $\boldsymbol{a}$, A confocal image showing part of the soma and dorsal dendrites of the cell. These receive several contacts from boutons that contain VGLUT2 (labeled with rhodamine) or CGRP (labeled with Pacific blue). Some of these contacts are indicated with arrows (VGLUT2) and arrowheads (GRP). The inset (which corresponds to the area outlined by the box) shows the same region scanned after the section had been further incubated in anti-VGLUT1 (VG1), which was also revealed with Pacific blue. The two profiles indicated with arrowheads are now Pacific blue labeled, indicating that they are VGLUT1 immunoreactive. $\boldsymbol{b}$ - $\boldsymbol{e}$, Part of a dorsal dendrite of this cell receives several contacts from VGLUT2 boutons (some shown with arrows) and (GRP boutons (some shown with arrowheads). All images were projections of three optical sections at 0.5 $\mu \mathrm{m} z$-spacing. Scale bar, $10 \mu \mathrm{m}$.

Table 2. The density of contacts from different types of bouton on the NK1r-immunoreactive lamina III neurons

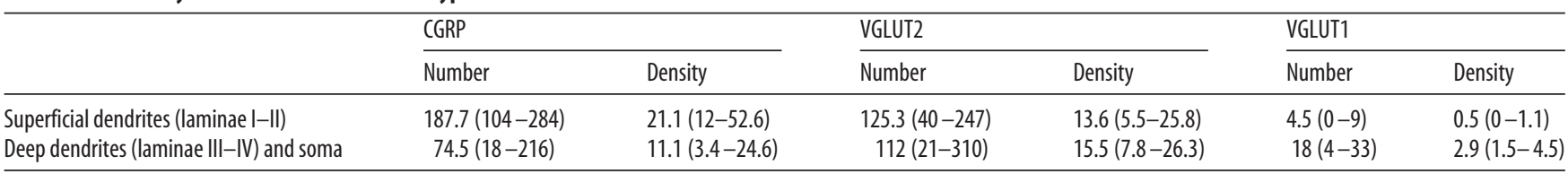

Mean numbers of different types of bouton that contacted the 15 lamina III NK1r cells, together with the density of contacts (per $1000 \mu \mathrm{m}^{2}$ of cell surface). Ranges are given in brackets. VGLUT2 boutons are those that showed strong VGLUT2 labeling and lacked CGRP and VGLUT1.

from CGRP and VGLUT1 boutons per unit length of dendrite. For CGRP, there were 17.4 (11.1-28) and 12.4 (3.1-27.9) contacts $/ 100 \mu \mathrm{m}$ for superficial and deep dendrites, respectively. The corresponding values for VGLUT1 were $0.4(0-1.1)$ and 3.7 (26.7) contacts/100 $\mu \mathrm{m}$.

\section{PPD expression by VGLUT2 boutons that contacted large NK1r neurons in laminae I and III}

The main aim of this part of the study was to identify VGLUT2 boutons that contacted large NK1r-positive lamina III neurons and determine the proportion of these that were PPD immunoreactive. To exclude peptidergic and low-threshold myelinated primary afferents, both of which can show low levels of VGLUT2 immunoreactivity, we only sampled those VGLUT2 ${ }^{+}$boutons that lacked CGRP and VGLUT1. Between 97 and 251 (mean 164) such boutons were identified in contact with the $15 \mathrm{NK} 1 \mathrm{r}$ lamina III cells that were analyzed, and PPD immunoreactivity was present in 58\% (range $41-68 \%$ ) of these (Fig. 2; Table 3). For VGLUT2 boutons that contacted deep dendrites and cell bodies the proportion that contained PPD was 63\% (range 39-76\%), while for those contacting superficial dendrites the proportion was 51\% (range $46-71 \%$ ), and these values differed significantly (Mann-Whitney $U$ test, $p<0.01$ ). As reported previously (Marvizón et al., 2009), we observed some boutons that were labeled with both PPD and CGRP, but these were never seen in contact with the lamina III NK1r projection cells.

Analysis of the general population of VGLUT2 boutons revealed that $6.5 \%$ (range $4-10 \%$ ) of those that were selected in laminae I-II and $4.7 \%$ (range $4-6 \%$ ) of those selected in laminae III-IV were PPD immunoreactive (Table 3). The mean $z$-axis lengths of PPD ${ }^{+}$and $\mathrm{PPD}^{-}$VGLUT2-immunoreactive boutons did not differ in laminae I-II (1.64 and $1.76 \mu \mathrm{m}$, respectively) whereas for laminae III-IV the mean length of the PPD ${ }^{+}$boutons $(2.02 \mu \mathrm{m})$ was significantly larger than that of the $\mathrm{PPD}^{-}$boutons $(1.62 \mu \mathrm{m})(p<0.01$; Mann-Whitney $U$ test $)$. This indicates that the selection process was not biased for the VGLUT2 boutons in laminae I-II, but that it was biased toward the $\mathrm{PPD}^{+}$boutons in laminae III-IV, as these were on average $1.25 \times$ longer in the $z$-axis. Since the extent of this bias is directly related to the difference in $z$-axis length, we estimate that the true proportion of VGLUT2 nonprimary boutons that express PPD in laminae III-IV is $3.7 \%$, assuming that the "corrected" number of PPD ${ }^{+}$ boutons in each sample would have been $0.8(1.62 / 2.02)$ of the actual number, with the same number of $\mathrm{PPD}^{-}$boutons being included. Two-way ANOVA on ranks with post hoc all-pairwise multiple comparisons (Holm-Sidak method) revealed that in both superficial and deep regions, the proportion of VGLUT2 boutons immunoreactive for PPD was significantly higher for those contacting lamina III NK1r cells than for those in the general population $(p<0.001)$ (Table 3$)$.

To test whether selective innervation by $\mathrm{PPD}$-containing glutamatergic terminals was a general feature of NK1r-expressing projection neurons, we also analyzed 15 large NK1r-positive neurons in lamina I, all of which are projection cells (Al Ghamdi et al., 2009). Although these also received some contacts from PPDcontaining VGLUT2 boutons (Fig. 3), these constituted only 

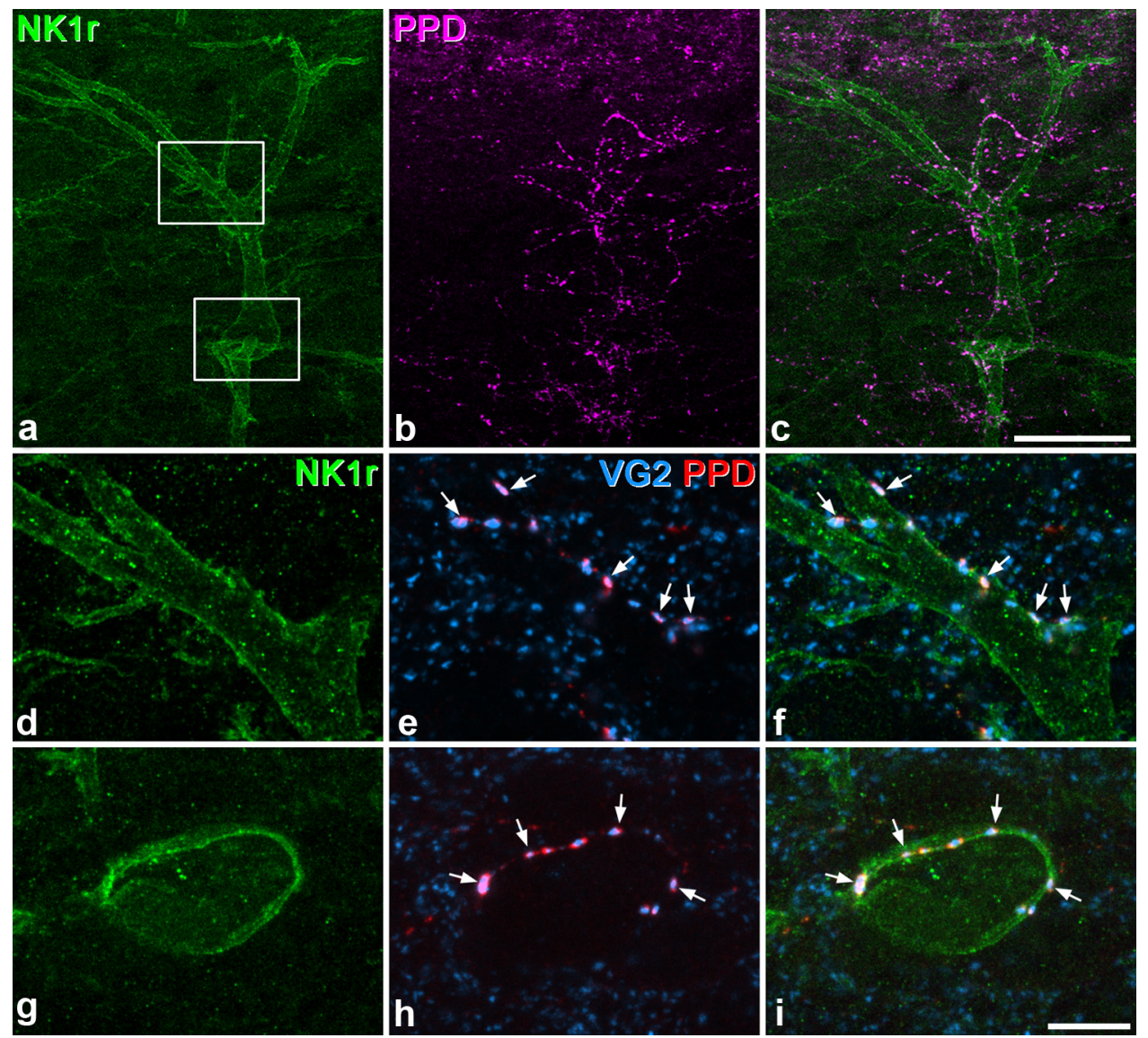

Figure 2. Contacts between glutamatergic axons that contain PPD and a lamina III neuron that expresses the NK1r. $\boldsymbol{a}-\boldsymbol{c}$, Low-magnification image through the soma and dorsal dendrites of the lamina III cell, showing its association with PPD axons. The two areas in boxes are shown at higher magnification in subsequent parts. $\boldsymbol{d}-\boldsymbol{f}$, The region in the upper box in $\boldsymbol{a}$. This part of the dorsal dendrite receives several contacts from boutons that are labeled with both PPD (red) and VGLUT2 (VG2, blue), and therefore appear magenta (some marked with arrows). Note that most VGLUT2 boutons in this field are not PPD immunoreactive. $\boldsymbol{g}-\boldsymbol{i}$, Several axosomatic contacts from boutons labeled with both PPD and VGLUT2 are visible (some marked with arrows). Images are from projections of $22(\boldsymbol{a}-\boldsymbol{c}), 3(\boldsymbol{d}-\boldsymbol{f})$, or $11(\boldsymbol{g}-\boldsymbol{i})$ optical sections at $0.5 \mu \mathrm{m} z$-spacing. Scale bars: (in c) $\boldsymbol{a}-\boldsymbol{c}, 50 \mu \mathrm{m}$; (in $\boldsymbol{i}) \boldsymbol{d}-\boldsymbol{i}, 10 \mu \mathrm{m}$.

Table 3. Percentages of different types of VGLUT2-positive boutons that were PPD-immunoreactive

\begin{tabular}{|c|c|c|}
\hline & $\begin{array}{l}\text { Number of VGLUT2 }{ }^{+} \\
\text {boutons }\end{array}$ & $\begin{array}{l}\text { \% VGLUT2 that were } \\
\text { PPD }^{+}\end{array}$ \\
\hline \multicolumn{3}{|l|}{$\begin{array}{l}\text { VGLUT2 boutons contacting lamina III } \\
\text { NK1r neurons }\end{array}$} \\
\hline Laminae I-II & $60.1(25-112)$ & $51.2(39.4-76)$ \\
\hline Laminae III-IV & $103.7(34-211)$ & $62.5(46.2-70.6)$ \\
\hline Total & $163.7(97-251)$ & $57.8(41.3-67.7)$ \\
\hline \multicolumn{3}{|l|}{$\begin{array}{l}\text { General population of VGLUT2 } \\
\text { boutons }\end{array}$} \\
\hline Laminae I-II & $100(100)$ & $6.5(4-10)$ \\
\hline Laminae III-IV & $100(100)$ & $4.7(4-6)$ \\
\hline $\begin{array}{l}\text { VGLUT2 boutons contacting lamina I NK1r } \\
\text { neurons }\end{array}$ & $129(73-184)$ & $10.8(5.7-14.9)$ \\
\hline
\end{tabular}

The mean numbers of VGLUT2 boutons and the percentage that were PPD immunoreactive for each analysis are shown in the second and third columns, with the ranges in brackets. The boutons in contact with the two different types of NK1r neuron were analyzed on 15 cells in each case. VGLUT2 boutons in the general population were selected from six sections (two from each of three rats) with 100 boutons per region analyzed on each section. Only VGLUT2 boutons that lacked CGRP or VGLUT1 were included in this analysis.

$11 \%$ of the VGLUT2 ${ }^{+}$boutons in contact with these cells. This was significantly different from the corresponding value for the lamina III NK1r cells $(p<0.001$; Mann-Whitney $U$ test $)$.

\section{Combined confocal and electron microscopy}

Twenty-two contacts between VGLUT2-immunoreactive boutons and the lamina III NK1r cell could be identified with the EM in the tissue reacted for NK1r, VGLUT2, and CGRP. Identification was achieved by comparing the location of DAB-positive profiles in low-magnification electron micrographs with rhodaminelabeled (VGLUT2 immunoreactive) profiles in corresponding optical sections from the confocal image stack. The dendritic shafts of the NK1r neuron were readily identified with the EM based on their location, size, and shape, even though the NK1r, which outlined them, was not revealed with DAB (Fig. 4). At 20 of the 22 contacts involving VGLUT2-immunoreactive boutons an asymmetrical synapse could be identified (Fig. $4 g-i$ ). Although the CGRP was not revealed with DAB, CGRP-immunoreactive boutons that contacted the NK1r cell could also be recognized based on their location (Fig. $4 c-e$ ). Fourteen CGRP boutons that contacted the cell were identified in this way, and all were found to contain dense-cored vesicles and to form asymmetrical synapses with the NK1r neuron (Fig. $4 f$ ).

The other cell examined with the EM was from tissue that had been reacted with NK1r, PPD, and VGLUT2. Although in this case all VGLUT2 boutons contained DAB reaction product, it was possible to identify those that were also PPD immunoreactive, based on the colocalization of PPD and VGLUT2 immunoreactivity seen in the confocal images (Fig. 5a). Twenty-three VGLUT2 boutons that contacted the cell were found with the EM, and 13 of these were also PPD immunoreactive. Synapses, all of which were asymmetrical, were identified at 20 of these contacts ( 10 of the 13 at which the bouton was PPD immunoreactive and all 10 of those at which the bouton lacked PPD) (Fig. 5). In 

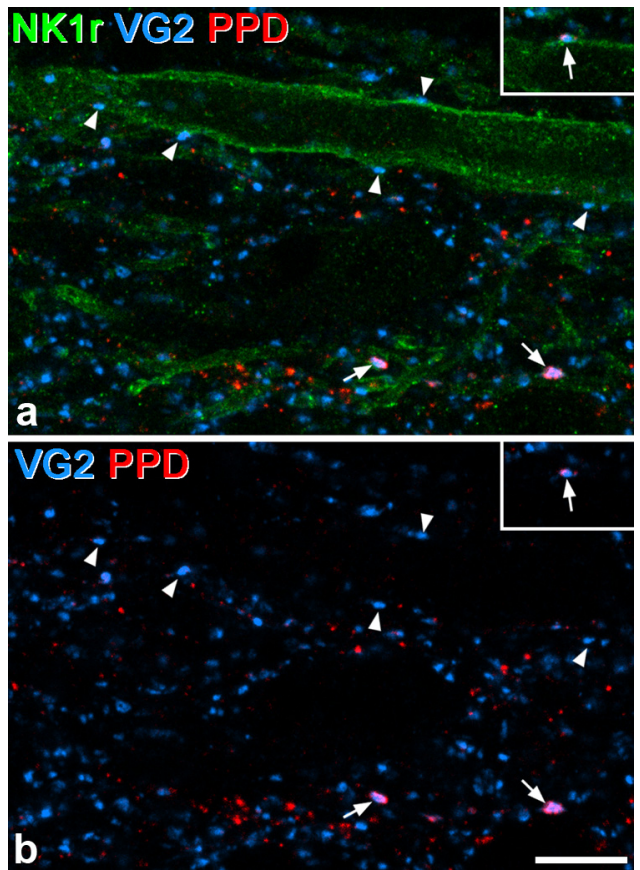

Figure 3. VGLUT2 contacts on a large NK1r-immunoreactive neuron in lamina I. $\boldsymbol{a}$, A proximal dendrite of the cell receives several contacts from VGLUT2-immunoreactive (VG2) boutons that lack PPD (arrowheads). Arrows indicate two nearby boutons that contain both VGLUT2 and PPD. The inset shows a contact that this dendrite received from a PPD ${ }^{+} / \mathrm{VGLUT2}^{+}$bouton at a different depth within the section (arrow). $\boldsymbol{b}$, The same field scanned to reveal only VGLUT2 and PPD shows that the great majority of VGLUT2 boutons in this region do not contain PPD. Projections of four (main image) or two (inset) optical sections at $0.5 \mu \mathrm{m} z$-spacing. Scale bar, $10 \mu \mathrm{m}$.

addition, we identified 12 asymmetrical synapses that the NK1r cell received from axonal boutons that were not labeled with DAB (and which were neither VGLUT2 nor PPD immunoreactive in the confocal image stacks), and which contained at least five dense-cored vesicles. Since these were found close to boutons with strong VGLUT2 immunoreactivity, it is unlikely that the lack of VGLUT2 staining represented a false negative result. The lamina III NK1r cells are known to receive a high density of synaptic input from peptidergic primary afferents (Naim et al., 1997), and it is likely that the VGLUT2-negative boutons with dense core vesicles were derived from these afferents.

The lengths of the synapses on both cells were measured from the electron micrographs and are shown in Figure 6. Lengths of the 40 synapses associated with VGLUT2 boutons ranged from 0.2 to $1.1 \mu \mathrm{m}$ (mean $0.55 \mu \mathrm{m}$ ) We compared the lengths of synapses formed by CGRP-immunoreactive boutons on the first cell with those formed by VGLUT2-negative boutons with dense cored vesicles on the second cell and found that these did not differ significantly (CGRP: mean $0.88 \pm 0.26 \mu \mathrm{m}, n=14$; VGLUT2 $^{-}$: mean $1.19 \pm 0.62 \mu \mathrm{m}, n=12 ; p=0.1, t$ test). Since the latter two groups are both likely to originate from peptidergic primary afferents, we pooled them and compared them to the synapses formed by VGLUT2-immunoreactive boutons on the two cells (Fig. 6). This showed a highly significant difference (VGLUT2-positive: mean $0.55 \pm 0.24 \mu \mathrm{m}, n=40$; peptidergic afferent: mean $1.02 \pm 0.48 \mu \mathrm{m}, n=26 ; p<0.001, t$ test $)$.

\section{Discussion}

The main findings of this study are that NK1r-expressing projection neurons in lamina III have numerous contacts from VGLUT2-immunoreactive boutons, which are distributed evenly across their dendritic trees, and that $>50 \%$ of these contain the dynorphin precursor PPD. The great majority of contacts were associated with synapses, although these were significantly smaller than the synapses formed by peptidergic primary afferents.

\section{Sources of glutamatergic input to lamina III NK1r projection neurons}

The lamina III projection neurons are densely innervated by peptidergic primary afferents (Naim et al., 1997), all of which should be CGRP immunoreactive (Ju et al., 1987). The density of contacts from CGRP boutons seen here was very similar to that reported by Naim et al. (1997) for substance P-immunoreactive boutons (mean values for superficial and deep dorsal dendrites: 18.8 and 13.2 contacts $/ 100 \mu \mathrm{m}$, respectively), consistent with the finding by Naim et al. (1997) that $95 \%$ of the substance P boutons contacting the cells were of primary afferent origin. This suggests that only peptidergic afferents that contain substance $P$ innervate these cells.

We have previously shown that these neurons receive some synapses on their deep dendrites from low-threshold myelinated afferents, which were identified with cholera toxin B subunit (CTb) injected into a peripheral nerve (Naim et al., 1998). All low-threshold myelinated afferents that are transganglionically labeled with CTb express high levels of VGLUT1 (Todd et al., 2003), and the only other source of VGLUT1-containing boutons in this region is the corticospinal tract (Casale et al., 1988; Alvarez et al., 2004). Our finding that the density of contacts from VGLUT1 boutons was very low in the superficial laminae, and that the density on deep dendrites was no higher than that of CTb-labeled primary afferent boutons (5.9 \pm 2 contacts/100 $\mu \mathrm{m})$ (Naim et al., 1998) suggests that these cells do not receive significant input from corticospinal axons.

Boutons belonging to peptidergic and low-threshold myelinated afferents can express low levels of VGLUT2 (Todd et al., 2003; Alvarez et al., 2004; Persson et al., 2006), and we therefore immunostained for both CGRP and VGLUT1 to exclude any of these from the population identified with VGLUT2 antibody. Although nonpeptidergic $\mathrm{C}$ nociceptors, which bind the lectin BS-IB4, also show low levels of VGLUT2 (Todd et al., 2003), these afferents do not innervate the lamina III projection neurons (Sakamoto et al., 1999).

Seal et al. (2009) identified a population of C low-threshold mechanoreceptive afferents (C-LTMRs) in mice, which express VGLUT3 and terminate in lamina IIi. We have found that although there is a plexus of VGLUT3-immunoreactive boutons in this region in the rat, many of these express VGLUT1 (A.J. Todd, unpublished observations), and so may originate from myelinated afferents. It is therefore not possible to assess the extent of any input to the lamina III projection neurons from C-LTMRs.

The cells received many contacts from VGLUT2-immunoreactive boutons, most of which were associated with synapses. The main source of these boutons is likely to be local excitatory neurons, which express high levels of VGLUT2 and generate substantial local axonal arbors (Todd et al., 2003; Schneider and Walker, 2007; Yasaka et al., 2010). However, there may also be a contribution from brainstem neurons with descending axons, and some of those in lamina I could originate from nonpeptidergic A $\delta$ nociceptors (Naim et al., 1998; Todd et al., 2003). 

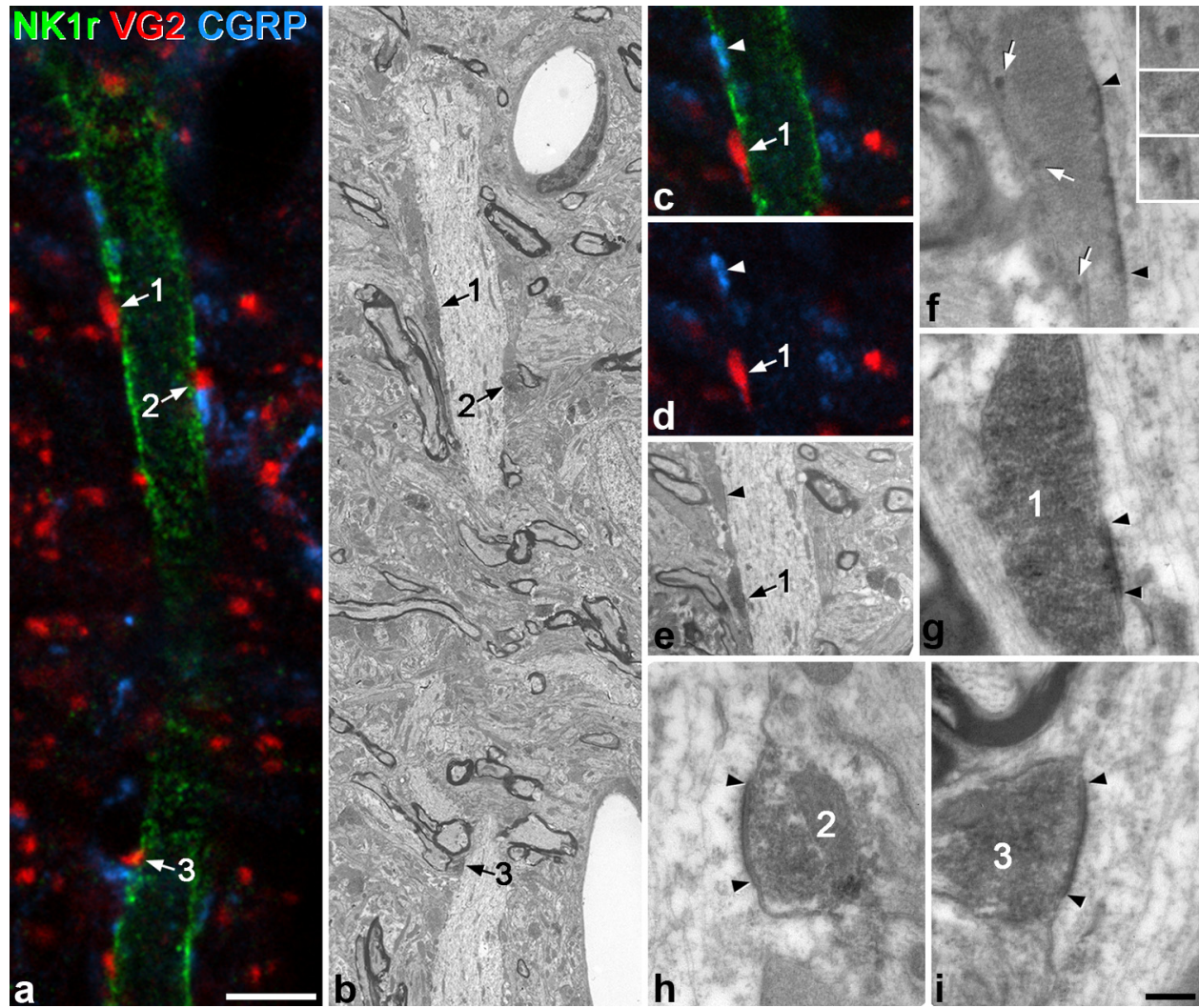

Figure 4. Synapses from boutons that contain VGLUT2 (VG2) or CGRP on the dorsal dendrite of a lamina III neuron with the NK1r, revealed with combined confocal and electron microscopy. $\boldsymbol{a}$, Single confocal optical section through the dorsal dendrite of the cell scanned to reveal NK1r (green), VGLUT2 (red), and CGRP (blue). Three contacts from VGLUT2-immunoreactive boutons are indicated with numbered arrows. $\boldsymbol{b}$, An electron micrograph from an ultrathin section through the cell at a level corresponding to the confocal image in $\boldsymbol{a}$. The dendritic shaft is visible, and the three VGLUT2 boutons, which are labeled with DAB are also seen. $\boldsymbol{c}, \boldsymbol{d}$, Confocal images from an optical section slightly deeper in the $z$-series than that shown in $\boldsymbol{a}$. These show the contact from one of the VGLUT2 boutons (numbered 1 in $\boldsymbol{a}$ ), as well as a contact from a nearby CGRP-immunoreactive bouton (arrowhead). $\boldsymbol{e}$, Electron micrograph from an ultrathin section corresponding to the region shown in c and $\boldsymbol{d}$. The VGLUT2 bouton is visible due to the DAB reaction product. The CGRP bouton is not labeled with DAB, but can be identified from its size and position relative to the dendrite and the VGLUT2 bouton. $\boldsymbol{f}$, High-magnification electron micrograph through the CGRP-immunoreactive bouton seen in $\boldsymbol{c}-\boldsymbol{e}$, which forms a synapse (between arrowheads) with the dendrite of the NK1r cell. Three dense-cored vesicles within the bouton are indicated with arrows, and these are shown at higher magnification in the insets. $\boldsymbol{g}-\boldsymbol{i}$, Synapses (between arrowheads) formed by the three VGLUT2 boutons. Scale bars: (in $\boldsymbol{a}$ ) $\boldsymbol{a}-\boldsymbol{e}, 5 \mu \mathrm{m}$; (in $\boldsymbol{i}$ ) $\boldsymbol{f}-\boldsymbol{i}, 0.25 \mu \mathrm{m}$.
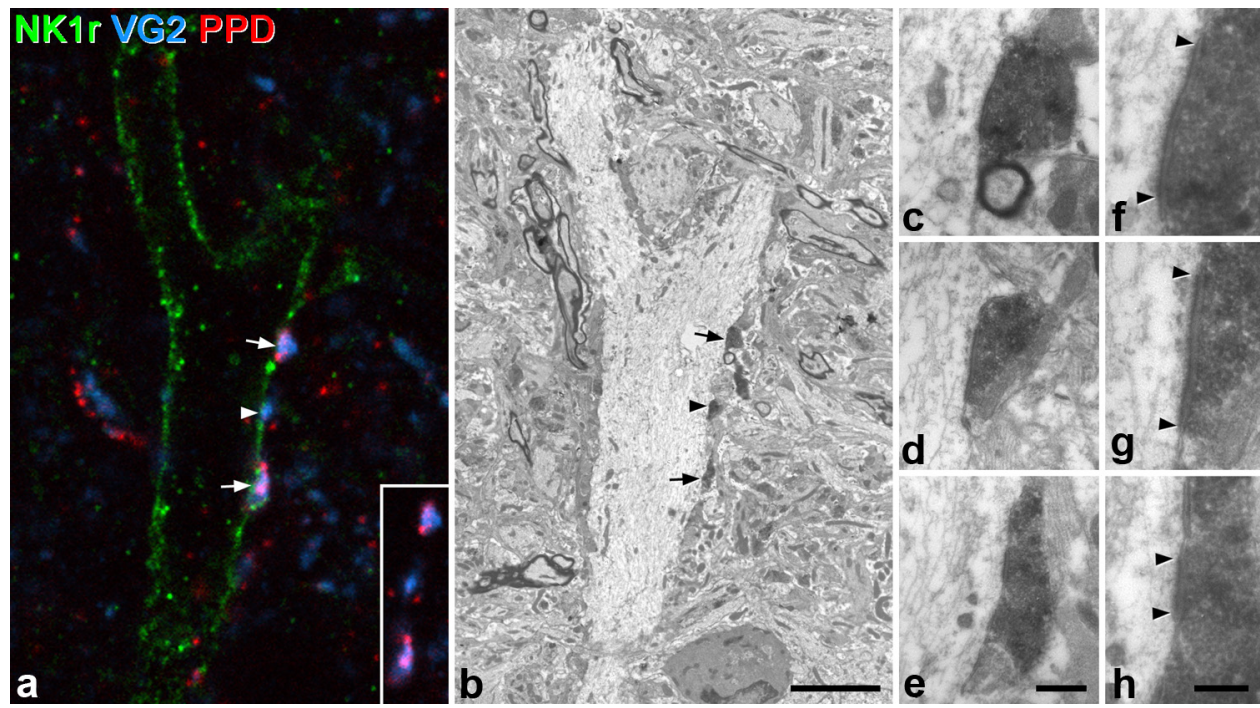

Figure 5. Synapses formed by boutons containing PPD and VGLUT2 (VG2) on the dorsal dendrite of a lamina III neuron with the NK1r, revealed with combined confocal and electron microscopy. $\boldsymbol{a}$, Single confocal optical section scanned to reveal NK1r (green), VGLUT2 (blue), and PPD (red). The dorsal dendrite of the NK1r-immunoreactive neuron receives three contacts from VGLUT2-immunoreactive boutons. Two of these also contain PPD, and therefore appear magenta (arrows), while the other one does not (arrowhead). The boutons are shown without NK1r immunostaining in the inset. $\boldsymbol{b}$, Low-magnification electron micrograph through the corresponding region of the dendrite. All three VGLUT2 boutons are visible, as the immunoperoxidase reaction was linked to the VGLUT2 antibody. $\mathbf{c}-\boldsymbol{e}, \boldsymbol{f}-\boldsymbol{h}$, Higher magnification views to show the presence of a synapse (between arrowheads) in each case. Scale bars: (in $\boldsymbol{b}) \boldsymbol{a}, \boldsymbol{b}, 5 \mu \mathrm{m}$; (in $\boldsymbol{e}$ ) $\boldsymbol{c}-\boldsymbol{e}, 0.5 \mu \mathrm{m}$; (in $\boldsymbol{h}) \boldsymbol{f}-\boldsymbol{h}, 0.25 \mu \mathrm{m}$. 


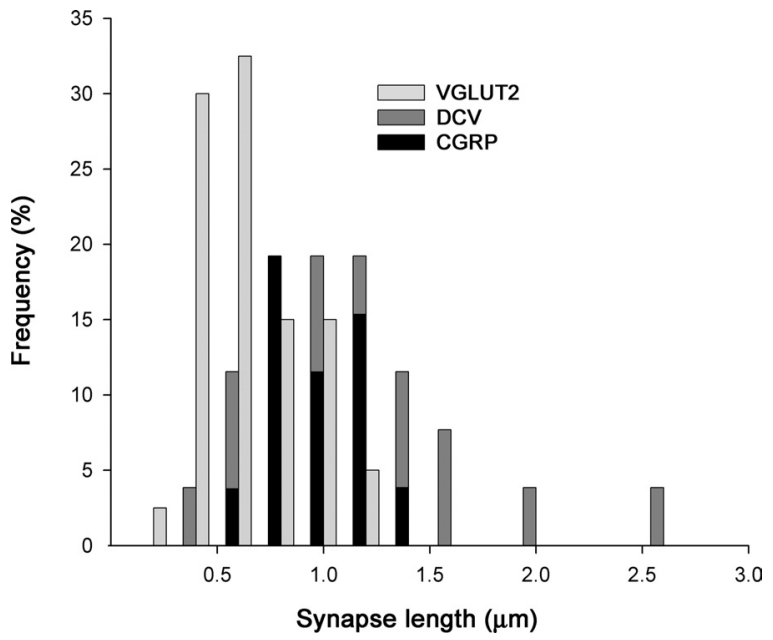

Figure 6. Synapse sizes for VGLUT2-immunoreactive and putative primary afferent boutons on lamina III NK1 receptor-immunoreactive cells. Frequency histogram showing the lengths of synapses from the VGLUT2 boutons identified on the two cells examined with electron microscopy (pale gray bars, $n=40$ ) and those of the putative primary afferents. The latter were identified by CGRP immunoreactivity in the first cell ( $n=14$, black bars) or the presence of dense cored vesicles in the second cell ( $n=12$, dark gray bars).

\section{Input from boutons that contain VGLUT2 and PPD}

A major finding of this study is that while PPD is expressed in $4-7 \%$ of all VGLUT2-immunoreactive boutons in laminae I-IV, it is present in $58 \%$ of those that contacted the lamina III projection neurons. This indicates a dramatic $(\sim 10$-fold $)$ enrichment of $\mathrm{PPD}^{+} / \mathrm{VGLUT}^{+}{ }^{+}$boutons, and demonstrates a highly selective targeting of these cells by axons of PPD-containing glutamatergic neurons. Both anti- and pro-nociceptive roles have been attributed to spinal dynorphin, through actions on $\kappa$-opioid and NMDA receptors, respectively (Laughlin et al., 1997; Tan-No et al., 2009). It is not known whether $\kappa$-opioid receptors are expressed by the lamina III projection neurons. However, the presence of asymmetrical synapses indicates that the input from $\mathrm{PPD}^{+} / \mathrm{VGLUT}^{+}$boutons operates through a glutamatergic mechanism, and dynorphin may enhance NMDA receptor activity at these synapses.

Potential sources of PPD boutons innervating the lamina III projection cells are primary afferents, descending axons, and local neurons. Although PPD is present in some CGRP-containing primary afferents (Marvizón et al., 2009), these did not contact the lamina III projection cells. Since all known classes of primary afferent, apart from A $\delta$ nociceptors, express very low levels of (or completely lack) VGLUT2 (Todd et al., 2003), it is unlikely that the $\mathrm{PPD}^{+} / \mathrm{VGLUT}^{+}{ }^{+}$boutons identified in this study were of primary afferent origin. PPD is expressed by several populations of neurons throughout the brainstem (Khachaturian et al., 1982; Vincent et al., 1982; Guthrie and Basbaum, 1984); however, the only regions reported to contain significant numbers of both PPD cells and spinally projecting neurons (Willis and Coggeshall, 2004) are the locus ceruleus and nucleus of the solitary tract (NTS). Axons descending from locus ceruleus do not contain dynorphin (Patel et al., 1997), and we have found that boutons in dorsal horn anterogradely labeled from NTS are not PPD immunoreactive (N. Baseer and A.J. Todd, unpublished observations).

It is therefore likely that the $\mathrm{PPD}^{+} / \mathrm{VGLUT}^{+}{ }^{+}$boutons that innervate the lamina III projection cells are derived exclusively from local spinal neurons. Within the spinal cord, PPDexpressing neurons are densely packed in superficial laminae and scattered throughout the deep dorsal horn (Khachaturian et al.,
1982; Vincent et al., 1982; Cruz and Basbaum, 1985; Standaert et al., 1986; Miller and Seybold, 1987; Ruda et al., 1988; Sardella et al., 2011a). Although most PPD cells in laminae I-II are GABAergic (inhibitory) interneurons, $\sim 30 \%$ of those in this region, and all of those in the deeper laminae, lack GABA and are thought to be glutamatergic (Sardella et al., 2011a). While most of these are presumably interneurons, some of those in lamina I are projection cells (Standaert et al., 1986; Nahin et al., 1989). It is likely that both interneurons and projection neurons contribute to the local plexus of $\mathrm{PPD}^{+} / \mathrm{VGLUT}^{+}$axons, since many lamina I projection neurons generate local axon collaterals (Szucs et al., 2010).

We have previously reported examples of selective targeting of projection cells by populations of inhibitory interneurons. Specifically, GABAergic neurons containing NPY innervate the lamina III NK1r projection cells (Polgár et al., 1999, 2011), while those that contain neuronal nitric oxide synthase (nNOS) innervate giant lamina I projection cells (Puskár et al., 2001). However, this is the first evidence that excitatory dorsal horn neurons also selectively innervate projection cells. In addition, this represents a far more dramatic enrichment $(\sim 10$ times greater than would occur by chance), compared with those found for NPYcontaining GABAergic boutons on these cells ( $\sim 2$ - to 3-fold enrichment in laminae I-II) (Polgár et al., 2011) or for $\mathrm{nNOS}^{+} /$ $\mathrm{GABA}^{+}$boutons on the giant lamina I cells (twofold enrichment) (Sardella et al., 2011b).

\section{Functional significance of primary and nonprimary glutamatergic input to lamina III projection neurons}

Our results indicate that these cells receive substantial excitatory input from local glutamatergic neurons, many of which express dynorphin. Since virtually all glutamatergic boutons that contact the lamina III projection cells are likely to have been detected by the combination of CGRP, VGLUT1, and VGLUT2 antibodies, we estimate that the VGLUT2 boutons account for $39 \%$ of those in laminae I-II and 53\% of those in laminae III-IV (Table 3). We previously reported that although most glutamatergic synapses on these projection cells were $<1 \mu \mathrm{m}$ long, some were highly elongated, and we predicted that these were associated with peptidergic primary afferents (Todd et al., 2009). The present findings confirm this, by showing that peptidergic afferent synapses are significantly larger than those formed by nonprimary $\left(\mathrm{VGLUT}^{+}{ }^{+}\right.$) boutons. Since these large synapses are associated with more postsynaptic glutamate receptors (Todd et al., 2009), they will presumably generate relatively large EPSPs, providing a highly secure monosynaptic input from nociceptive primary afferents.

Less is known about the functions of local glutamatergic neurons that innervate the lamina III projection cells. However, many of the dynorphin-containing cells are likely to be activated by noxious stimuli, since cells with PPD mRNA located throughout the dorsal horn express Fos after noxious stimulation (Noguchi et al., 1991). The lamina III projection neurons are therefore presumably activated by nociceptive primary afferents through both monosynaptic and polysynaptic pathways. We recently reported that excitatory interneurons in lamina II show firing patterns associated with A-type potassium $\left(I_{A}\right)$ currents (Yasaka et al., 2010). This suggests that these cells are normally inexcitable, but can undergo activity-dependent plasticity (Sandkühler, 2009), involving inactivation of these currents due to phosphorylation of channel proteins ( $\mathrm{Hu}$ et al., 2006). If dynorphincontaining excitatory neurons are among those possessing $I_{A}$ currents, acute noxious stimuli may normally activate the lamina III projection cells mainly through monosynaptic inputs. How- 
ever, prolonged noxious stimulation will suppress $I_{A}$ currents in the dynorphin neurons (Hu et al., 2006), leading to amplification of the polysynaptic nociceptive input to the projection cells. This, together with the activation of NMDA receptors by dynorphin released locally, could contribute to the development of inflammatory pain states.

\section{References}

Al Ghamdi KS, Polgár E, Todd AJ (2009) Soma size distinguishes projection neurons from neurokinin 1 receptor-expressing interneurons in lamina I of the rat lumbar spinal dorsal horn. Neuroscience 164:1794-1804.

Al-Khater KM, Kerr R, Todd AJ (2008) A quantitative study of spinothalamic neurons in laminae I, III, and IV in lumbar and cervical segments of the rat spinal cord. J Comp Neurol 511:1-18.

Alvarez FJ, Villalba RM, Zerda R, Schneider SP (2004) Vesicular glutamate transporters in the spinal cord, with special reference to sensory primary afferent synapses. J Comp Neurol 472:257-280.

Casale EJ, Light AR, Rustioni A (1988) Direct projection of the corticospinal tract to the superficial laminae of the spinal cord in the rat. J Comp Neurol 278:275-286.

Cruz L, Basbaum AI (1985) Multiple opioid peptides and the modulation of pain: immunohistochemical analysis of dynorphin and enkephalin in the trigeminal nucleus caudalis and spinal cord of the cat. J Comp Neurol 240:331-348.

Graham BA, Brichta AM, Callister RJ (2007) Moving from an averaged to specific view of spinal cord pain processing circuits. J Neurophysiol 98:1057-1063.

Grudt TJ, Perl ER (2002) Correlations between neuronal morphology and electrophysiological features in the rodent superficial dorsal horn. J Physiol 540:189-207.

Guillery RW (2002) On counting and counting errors. J Comp Neurol 447:1-7.

Guthrie J, Basbaum AI (1984) Colocalization of immunoreactive proenkephalin and prodynorphin products in medullary neurons of the rat. Neuropeptides 4:437-445.

Heinke B, Ruscheweyh R, Forsthuber L, Wunderbaldinger G, Sandkühler J (2004) Physiological, neurochemical and morphological properties of a subgroup of GABAergic spinal lamina II neurones identified by expression of green fluorescent protein in mice. J Physiol 560:249-266.

Hu HJ, Carrasquillo Y, Karim F, Jung WE, Nerbonne JM, Schwarz TL, Gereau RW 4th (2006) The kv4.2 potassium channel subunit is required for pain plasticity. Neuron 50:89-100.

Ju G, Hökfelt T, Brodin E, Fahrenkrug J, Fischer JA, Frey P, Elde RP, Brown JC (1987) Primary sensory neurons of the rat showing calcitonin gene-related peptide immunoreactivity and their relation to substance P-, somatostatin-, galanin-, vasoactive intestinal polypeptide- and cholecystokininimmunoreactive ganglion cells. Cell Tissue Res 247:417-431.

Kawamura Y, Fukaya M, Maejima T, Yoshida T, Miura E, Watanabe M, Ohno-Shosaku T, Kano M (2006) The CB1 cannabinoid receptor is the major cannabinoid receptor at excitatory presynaptic sites in the hippocampus and cerebellum. J Neurosci 26:2991-3001.

Khachaturian H, Watson SJ, Lewis ME, Coy D, Goldstein A, Akil H (1982) Dynorphin immunocytochemistry in the rat central nervous system. Peptides 3:941-954.

Laughlin TM, Vanderah TW, Lashbrook J, Nichols ML, Ossipov M, Porreca F, Wilcox GL (1997) Spinally administered dynorphin A produces longlasting allodynia: involvement of NMDA but not opioid receptors. Pain 72:253-260.

Lawson SN, Crepps BA, Perl ER (1997) Relationship of substance P to afferent characteristics of dorsal root ganglion neurones in guinea-pig. J Physiol 505:177-191.

Lee T, Kaneko T, Taki K, Mizuno N (1997) Preprodynorphin-, preproenkephalin-, and preprotachykinin-expressing neurons in the rat neostriatum: an analysis by immunocytochemistry and retrograde tracing. J Comp Neurol 386:229-244.

Llewellyn-Smith IJ, Martin CL, Fenwick NM, Dicarlo SE, Lujan HL, Schreihofer AM (2007) VGLUT1 and VGLUT2 innervation in autonomic regions of intact and transected rat spinal cord. J Comp Neurol 503:741-767.

Lu Y, Perl ER (2005) Modular organization of excitatory circuits between neurons of the spinal superficial dorsal horn (laminae I and II). J Neurosci 25:3900-3907.
Marshall GE, Shehab SA, Spike RC, Todd AJ (1996) Neurokinin-1 receptors on lumbar spinothalamic neurons in the rat. Neuroscience 72:255-263.

Marvizón JC, Chen W, Murphy N (2009) Enkephalins, dynorphins, and beta-endorphin in the rat dorsal horn: an immunofluorescence colocalization study. J Comp Neurol 517:51-68.

Miller KE, Seybold VS (1987) Comparison of met-enkephalin-, dynorphin A-, and neurotensin-immunoreactive neurons in the cat and rat spinal cords: I. Lumbar cord. J Comp Neurol 255:293-304.

Nahin RL, Hylden JL, Iadarola MJ, Dubner R (1989) Peripheral inflammation is associated with increased dynorphin immunoreactivity in both projection and local circuit neurons in the superficial dorsal horn of the rat lumbar spinal cord. Neurosci Lett 96:247-252.

Naim MM, Shehab SA, Todd AJ (1998) Cells in laminae III and IV of the rat spinal cord which possess the neurokinin-1 receptor receive monosynaptic input from myelinated primary afferents. Eur J Neurosci 10:3012-3019.

Naim M, Spike RC, Watt C, Shehab SA, Todd AJ (1997) Cells in laminae III and IV of the rat spinal cord that possess the neurokinin-1 receptor and have dorsally directed dendrites receive a major synaptic input from tachykinin-containing primary afferents. J Neurosci 17:5536-5548.

Noguchi K, Kowalski K, Traub R, Solodkin A, Iadarola MJ, Ruda MA (1991) Dynorphin expression and Fos-like immunoreactivity following inflammation induced hyperalgesia are colocalized in spinal cord neurons. Brain Res Mol Brain Res 10:227-233.

Patel R, Kerr R, Maxwell DJ (1997) Absence of co-localized glutamic acid decarboxylase and neuropeptides in noradrenergic axons of the rat spinal cord. Brain Res 749:164-169.

Persson S, Boulland JL, Aspling M, Larsson M, Fremeau RT Jr, Edwards RH, Storm-Mathisen J, Chaudhry FA, Broman J (2006) Distribution of vesicular glutamate transporters 1 and 2 in the rat spinal cord, with a note on the spinocervical tract. J Comp Neurol 497:683-701.

Polgár E, Shehab SA, Watt C, Todd AJ (1999) GABAergic neurons that contain neuropeptide $Y$ selectively target cells with the neurokinin 1 receptor in laminae III and IV of the rat spinal cord. J Neurosci 19:2637-2646.

Polgár E, Sardella TC, Watanabe M, Todd AJ (2011) A quantitative study of NPY-expressing GABAergic neurons and axons in rat spinal dorsal horn. J Comp Neurol 519:1007-1023.

Ptak K, Burnet H, Blanchi B, Sieweke M, De Felipe C, Hunt SP, Monteau R, Hilaire G (2002) The murine neurokinin NK1 receptor gene contributes to the adult hypoxic facilitation of ventilation. Eur J Neurosci 16:2245-2252.

Puskár Z, Polgár E, Todd AJ (2001) A population of large lamina I projection neurons with selective inhibitory input in rat spinal cord. Neuroscience 102:167-176.

Ruda MA, Iadarola MJ, Cohen LV, Young WS 3rd (1988) In situ hybridization histochemistry and immunocytochemistry reveal an increase in spinal dynorphin biosynthesis in a rat model of peripheral inflammation and hyperalgesia. Proc Natl Acad Sci U S A 85:622-626.

Sakamoto H, Spike RC, Todd AJ (1999) Neurons in laminae III and IV of the rat spinal cord with the neurokinin-1 receptor receive few contacts from unmyelinated primary afferents which do not contain substance $P$. Neuroscience 94:903-908.

Sandkühler J (2009) Models and mechanisms of hyperalgesia and allodynia. Physiol Rev 89:707-758.

Sardella TC, Polgár E, Garzillo F, Furuta T, Kaneko T, Watanabe M, Todd AJ (2011a) Dynorphin is expressed primarily by GABAergic neurons that contain galanin in the rat dorsal horn. Mol Pain 7:76.

Sardella TC, Polgár E, Watanabe M, Todd AJ (2011b) A quantitative study of neuronal nitric oxide synthase expression in laminae I-III of the rat spinal dorsal horn. Neuroscience 192:708-720.

Schneider SP, Walker TM (2007) Morphology and electrophysiological properties of hamster spinal dorsal horn neurons that express VGLUT2 and enkephalin. J Comp Neurol 501:790-809.

Seal RP, Wang X, Guan Y, Raja SN, Woodbury CJ, Basbaum AI, Edwards RH (2009) Injury-induced mechanical hypersensitivity requires C-low threshold mechanoreceptors. Nature 462:651-655.

Shrestha SS, Bannatyne BA, Jankowska E, Hammar I, Nilsson E, Maxwell DJ (2012) Excitatory inputs to four types of spinocerebellar tract neurons in the cat and the rat thoraco-lumbar spinal cord. J Physiol 590:1737-1755.

Standaert DG, Watson SJ, Houghten RA, Saper CB (1986) Opioid peptide immunoreactivity in spinal and trigeminal dorsal horn neurons projecting to the parabrachial nucleus in the rat. J Neurosci 6:1220-1226.

Szucs P, Luz LL, Lima D, Safronov BV (2010) Local axon collaterals of lam- 
ina I projection neurons in the spinal cord of young rats. J Comp Neurol 518:2645-2665.

Tan-No K, Takahashi H, Nakagawasai O, Niijima F, Sakurada S, Bakalkin G, Terenius L, Tadano T (2009) Nociceptive behavior induced by the endogenous opioid peptides dynorphins in uninjured mice: evidence with intrathecal $\mathrm{N}$-ethylmaleimide inhibiting dynorphin degradation. Int Rev Neurobiol 85:191-205.

Tiong SY, Polgár E, van Kralingen JC, Watanabe M, Todd AJ (2011) Galanin-immunoreactivity identifies a distinct population of inhibitory interneurons in laminae I-III of the rat spinal cord. Mol Pain 7:36.

Todd AJ (1997) A method for combining confocal and electron microscopic examination of sections processed for double- or triple-labelling immunocytochemistry. J Neurosci Methods 73:149-157.

Todd AJ (2010) Neuronal circuitry for pain processing in the dorsal horn. Nat Rev Neurosci 11:823-836.

Todd AJ, McGill MM, Shehab SA (2000) Neurokinin 1 receptor expression by neurons in laminae I, III and IV of the rat spinal dorsal horn that project to the brainstem. Eur J Neurosci 12:689-700.

Todd AJ, Puskar Z, Spike RC, Hughes C, Watt C, Forrest L (2002) Projection neurons in lamina I of rat spinal cord with the neurokinin 1 receptor are selectively innervated by substance P-containing afferents and respond to noxious stimulation. J Neurosci 22:4103-4113.
Todd AJ, Hughes DI, Polgár E, Nagy GG, Mackie M, Ottersen OP, Maxwell DJ (2003) The expression of vesicular glutamate transporters VGLUT1 and VGLUT2 in neurochemically defined axonal populations in the rat spinal cord with emphasis on the dorsal horn. Eur J Neurosci 17:13-27.

Todd AJ, Polgár E, Watt C, Bailey ME, Watanabe M (2009) Neurokinin 1 receptor-expressing projection neurons in laminae III and IV of the rat spinal cord have synaptic AMPA receptors that contain GluR2, GluR3 and GluR4 subunits. Eur J Neurosci 29:718-726.

Vincent SR, Hökfelt T, Christensson I, Terenius L (1982) Dynorphinimmunoreactive neurons in the central nervous system of the rat. Neurosci Lett 33:185-190.

Willis WD, Coggeshall RE (2004) Sensory mechanisms of the spinal cord, Vol 2. New York: Kluwer Academic.

Yasaka T, Kato G, Furue H, Rashid MH, Sonohata M, Tamae A, Murata Y, Masuko S, Yoshimura M (2007) Cell-type-specific excitatory and inhibitory circuits involving primary afferents in the substantia gelatinosa of the rat spinal dorsal horn in vitro. J Physiol 581:603-618.

Yasaka T, Tiong SY, Hughes DI, Riddell JS, Todd AJ (2010) Populations of inhibitory and excitatory interneurons in lamina II of the adult rat spinal dorsal horn revealed by a combined electrophysiological and anatomical approach. Pain 151:475-488. 